\title{
An unusual late complication in traumatic brain injury: Persistent dysphagia due to tracheoesophageal fistula
}

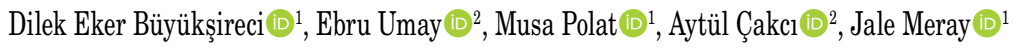 \\ ${ }^{1}$ Department of Physical Medicine and Rehabilitation, Medicine Faculty of Gazi University, Ankara, Turkey \\ ${ }^{2}$ Department of Physical Medicine and Rehabilitation, Ministry of Health Ankara Dışkapı Yıldırım Beyazıt Training and Research Hospital, Ankara, Turkey \\ Received: March 31, 2017 Accepted: September 12, 2017 Published online: May 07, 2018
}

\begin{abstract}
Traumatic brain injury (TBI), which leads to cognitive, physical, emotional and behavioral deficits according to the severity of trauma, is a disability with high morbidity and mortality. In addition to primary effects of TBI, direct trauma to the face, neck, and chest also contributes to increased morbidity. Recovery in swallowing functions in TBI patients is often parallel with recovery in functional daily activities. Herein, contrary to that expectation, we present a TBI case with persistent dysphagia symptoms lasting for even 1.5 years after trauma, despite the fact that she gained independence in dailylife activities under supervision.
\end{abstract}

Keywords: Dysphagia; tracheoesophageal fistula; tracheostomy cannula; traumatic brain injury.

Traumatic brain injury (TBI), which leads to cognitive, physical, emotional, and behavioral deficits depending on the severity of trauma, is a disability with high morbidity and mortality. In addition to primary effects of TBI, direct trauma to the face, neck and chest also contributes to increased morbidity. Dysphagia or swallowing disorder is a common complication for patients with neurological disorders such as TBI with an incidence as high as $93 \%$ in patients admitted to brain injury rehabilitation. ${ }^{[1,2]}$ Dysphagia in TBI associated with disorders in oral and pharyngeal phases of swallowing is due to diffuse axonal damage occurring in the brain. In particular, pharyngeal phase disorders can lead to life-threatening aspiration. Multiple surgical interventions, prolonged non-use of swallowing muscles due to unconsciousness, traumatic intubation in emergency conditions, and prolonged tracheostomy/intubation are additional risk factors for dysphagia in TBI patients. Dysphagia is seen usually in early-stage of TBI and improves within the first three months. ${ }^{[3]}$ Improvement in dysphagia is parallel to functional recovery.

Tracheoesophageal fistula (TEF) is an abnormal communication between the trachea and esophagus.
Tracheoesophageal fistula can be due to endotracheal intubation and tracheostomy. ${ }^{[4]}$ Acquired TEF usually develops after 15 to 200 days of mechanical ventilation. ${ }^{[5]}$ In such cases, tracheostomy is an independent risk factor for dysphagia.

Herein, we present a TBI case with persistent dysphagia symptoms lasting for even 1.5 years after trauma, despite the fact that she gained independence in dailylife activities under supervision.

\section{CASE REPORT}

A 16-year-old girl was admitted to our clinic for improving motor skills in the upper extremities fine motor control and mobilization. She had a TBI due to a traffic accident 1.5 years ago. At that time, cranial computed tomography (CT) of the patient revealed parenchymal hemorrhage with diffuse axonal damage in bilateral parietal gray matter, left thalamus and cerebellum. No additional trauma was detected in the chest radiograph and CT. She was intubated at that time and hospitalized in the intensive care unit for approximately 70 days and mechanical ventilation was also performed. She was, then, transferred to another

Corresponding author: Dilek Eker Büyükşireci, MD. Gazi Üniversitesi Tıp Fakültesi Fiziksel Tıp ve Rehabilitasyon Anabilim Dalı, 06500 Beşevler, Ankara, Turkey. e-mail: dilekeker55@gmail.com 


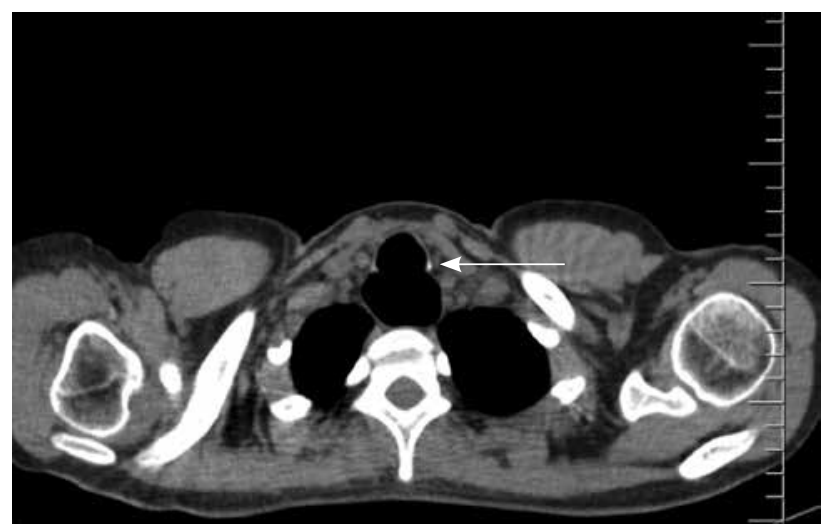

Figure 1. A $23 \times 13 \mathrm{~mm}$ fistula between trachea and esophagus.

rehabilitation clinic with gastrostomy tube for feeding, spontaneous respiration via tracheostomy cannula, and in state of Glasgow Coma Scale score 8. The patient had intense vomiting and recurrent aspiration pneumonia at this time, and fiberoptic endoscopic evaluation (FEE) and abdominal ultrasonography (USG) for the diagnosis were performed. Fiberoptic endoscopic evaluation did not show any residue, aspiration of liquid, semi-solid and solid foods; however, USG demonstrated acute cholecystitis and she was operated for this condition. Postoperatively, vomiting decreased, tracheostomy cannula was removed, oral feeding was started, and the patient began to walk independently following rehabilitation. After one year, she was still complaining of vomiting and coughing during the meal, although the functional gains were still present. The patient had a history of taking antibiotics with the cause of pneumonia two times during this period, on which the lung examination was performed. Respiratory sounds and chest radiographs were normal. There was no sign of aspiration in bedside screen test and FEE showed no residue or aspiration. Thoracic CT for the assessment of esophageal phase of swallowing revealed the presence of a fistula between the trachea and esophagus at the mediastinum entrance (Figure 1). She was operated for fistula and postoperatively, symptoms of coughing and vomiting during feeding disappeared. Therefore, swallowing dysfunction was thought to be due to TEF.

\section{DISCUSSION}

Dysphagia due to oral and pharyngeal phase disorders is frequently seen in TBI patients. The most common cause of dysphagia in these patients have been reported as premature escape due to reduction in cognitive function, and residue and aspiration due to decreased laryngeal elevation. ${ }^{[6,7]}$ In recent years, due to increasing interest to dysphagia, it is recognized early with bedside screening tests and FEE, and mortality related to dysphagia is being reduced with diet and feeding modification. ${ }^{[2]}$ In our case, post-traumatic gastrostomy tube for insufficient swallowing functions was performed early. As parallel to the cognitive and physical recovery of the patient, she was controlled for dysphagia and full oral intake was allowed. However, in our case, symptoms of coughing and vomiting continued during feeding and we thought that this condition was associated with the presence of a TEF, as swallowing fully recovered postoperatively. Tracheoesophageal fistulas are characterized by the presence of an abnormal relationship between the respiration and the digestive tract. The consequences of permanent contamination of the pulmonary system by food containing digestive secretions can be very serious and fatal. Acquired TEFs are rare complications and intubation complication with cuff-related tracheal injury is the most common etiology of non-malignant TEFs. ${ }^{[8]}$ Although the introduction of high volume and low pressure endotracheal tube cuffs reduce the incidence of this complication; the main cause of benign TEFs is prolonged intubation and tracheostomy. ${ }^{[9]}$

Jiaxin et al. ${ }^{[10]}$ presented a 57 -year-old woman with a myasthenic crisis. She had nasal-sounding speech, difficulty in chewing, disphagia, respiratory difficulties, and limb weakness, for which she received tracheal intubation and mechanic ventilation. Tracheostomy was performed 10 days later and after 27 days of treatment, the tracheal intubation catheter and nasogastric tube were removed. Her chewing, respiration, and speech were restored well, although she still coughed when drinking and experienced dysphagia. A thoracic CT scan revealed a TEF, $2 \mathrm{~cm}$ in diameter, and the reason of dysphagia was considered to be TEF associated with long-term tracheostomy. In our case, tracheostomy cannula was present for about a year. There was no inflammatory/ infectious disease, direct trauma, no history of surgical intervention leading to the development of TEF. Therefore, dysphagia was thought as the reason of TEF.

In conclusion, TEFs are uncommon and easily missed complications. As this condition has typical clinical characteristics that are similar to those of the primary disease, diagnosticians may fail to consider this rare condition in the differential diagnosis. 


\section{Declaration of conflicting interests}

The authors declared no conflicts of interest with respect to the authorship and/or publication of this article.

\section{Funding}

The authors received no financial support for the research and/or authorship of this article.

\section{REFERENCES}

1. Mandaville A, Ray A, Robertson H, Foster C, Jesser C. A retrospective review of swallow dysfunction in patients with severe traumatic brain injury. Dysphagia 2014;29:310-8.

2. Howle AA, Baguley I, Brown J, Curr L. Management of dysphagia following traumatic brain injury. Phys Med Rehabil Rep 2014;2:219-30.

3. Morgan A, Ward E, Murdoch B, Bilbie K. Acute characteristics of pediatric dysphagia subsequent to traumatic brain injury: videofluoroscopic assessment. J Head Trauma Rehabil 2002;17:220-41.
4. Shen KR, Allen MS, Cassivi SD, Nichols FC, Wigle DA, Harmsen WS, et al. Surgical management of acquired nonmalignant tracheoesophageal and bronchoesophageal fistulae. Ann Thorac Surg 2010;90:914-9.

5. Rao SV, Boralkar AK, Jirvankar PS, Sonavani MV, Kaginalkar VR, Chinte C. Tracheoesophageal Fistula following Endotracheal Intubation for Organophosphorus Poisoning. J Assoc Physicians India 2016;64:84-85.

6. Terré R, Mearin F. Videofluoroscopy quantification of laryngotracheal aspiration outcome in traumatic brain injury-related oropharyngeal dysphagia]. Rev Esp Enferm Dig 2007;99:7-12.

7. Terré R, Mearin F. Prospective evaluation of oro-pharyngeal dysphagia after severe traumatic brain injury. Brain Inj 2007;21:1411-7.

8. Reed MF, Mathisen DJ. Tracheoesophageal fistula. Chest Surg Clin N Am 2003;13:271-89.

9. Paraschiv M. Tracheoesophageal fistula--a complication of prolonged tracheal intubation. J Med Life 2014;7:516-21.

10. Jiaxin C, Jingjing L, Kai Z, Zhou Z, Weibin L, Haiyan W, et al. Tracheoesophageal Fistula Caused by Tracheostomy in a Patient with Myasthenia Gravis after a Myasthenic Crisis. Front Neurol 2017;8:217. 